\title{
Optically detected flip-flops between different spin ensembles in diamond
}

\author{
Sergei Masis, Sergey Hazanov, Nir Alfasi, Oleg Shtempluck, and Eyal Buks \\ Andrew and Erna Viterbi Department of Electrical Engineering, Technion, Haifa 32000 Israel
}

(Dated: March 17, 2021)

\begin{abstract}
We employ the technique of optical detection of magnetic resonance to study dipolar interaction in diamond between nitrogen-vacancy color centers of different crystallographic orientations and substitutional nitrogen defects. We demonstrate optical measurements of resonant spin flips-flips (second Larmor line), and flip-flops between different spin ensembles in diamond. In addition, the strain coupling between the nitrogen-vacancy color centers and bulk acoustic modes is studied using optical detection. Our findings may help optimizing cross polarization protocols, which, in turn, may allow improving the sensitivity of diamond-based detectors.
\end{abstract}

PACS numbers:

\section{INTRODUCTION}

A color center in diamond composed of a substitutional nitrogen and a vacancy in the crystal lattice (NV) [1] draws lately a considerable attention, particularly in the negatively charged state $\left(\mathrm{NV}^{-}\right)$. The $\mathrm{NV}^{-}$electronic spin state can be polarized and read out with light. Dense ensembles of $\mathrm{NV}^{-}$centers were demonstrated to be applicable for magnetometry [2], classical [3] and quantum [4 7] information storage, and recently, a maser implementation [8].

Dipolar coupling affecting a given $\mathrm{NV}^{-}$in a diamond crystal is commonly dominated by other $\mathrm{NV}^{-} \mathrm{s}$, having either parallel or non-parallel lattice orientation, and by substitutional nitrogen (P1) defects, having a density typically higher by at least an order of magnitude. The interaction between the ensembles might be a quantum resource [9] or a source of decoherence [10].

Spontaneous, i.e. phonon assisted, spin flip-flops between ensembles (when one of the spins changes from high to low energy states, and the other changes in the opposite direction), were demonstrated before [11 14]. In this work we demonstrate direct and unambiguous stimulated flip-flop interaction between the parallel and the non-parallel $\mathrm{NV}^{-}$ensembles or the $\mathrm{P} 1$ ensemble. A higher order process involving five spins interaction is reported elsewhere [15]. In addition, we observe dipolar spin-spin interaction [16] at double the Larmor frequency inside a single $\mathrm{NV}^{-}$ensemble parallel to the external magnetic field. The increased sensitivity of our setup is attributed to a combination of high $\mathrm{NV}^{-}$density, low temperature and lock-in amplification.

In the context of sensing, our results may be described as optically detected electron-electron double resonance [17] (ELDOR, also known as DEER). Unlike the traditional, microwave (MW) cavity based ELDOR, optical detection allows increased sensitivity, wide range of MW frequencies and higher spacial resolution. In the context of hyperpolarization [18], our method is closely related to dynamic nuclear polarization [19] (DNP), where in the role of slow nuclear spins are the electronic spins of the $\mathrm{P} 1$, and in the role of fast electron spins are the optically polarized $\mathrm{NV}^{-}$. Hyperpolarized

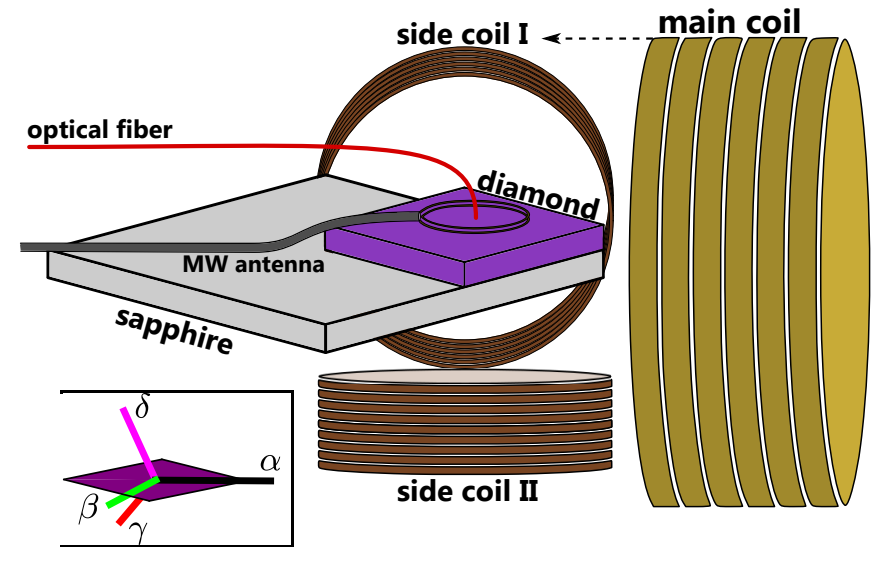

FIG. 1: Experimental setup. Radio frequency radiation is introduced by a flexible coaxial cable with a MW loop antenna termination pressed against the (110) diamond face. A multi-mode optical fiber normal to the diamond wafer, and positioned in the center of the loop antenna, is used both to illuminate and to collect PL. A superconducting solenoid (main coil) produces strong longitudinal magnetic field $B_{\|}$, aligned in the direction of diamond $\alpha$ vector by two smaller superconducting coils. Inset: diamond crystallographic directions. Vectors $\alpha([1 \overline{1} \overline{1}])$ and $\beta([\overline{1} 1 \overline{1}])$ are in the (110) plane, nominally parallel to the substrate surface depicted by the purple rectangular patch. Vectors $\gamma([\overline{1} \overline{1} 1])$ and $\delta([111])$ are not in the (110) surface and are mirrored by it.

P1 then might serve as a low noise spin bath for the $\mathrm{NV}^{-}$, or as a target spin ensemble on its own right, e.g. for applications of P1 maser.

\section{ODMR MEASUREMENTS}

When hyperfine interaction is disregarded, the $\mathrm{NV}^{-}$ground state spin triplet Hamiltonian becomes [26, 27 .

$$
\frac{\mathcal{H}_{\mathrm{NV}}}{\hbar}=\frac{D_{\mathrm{NV}} S_{z}^{2}}{\hbar^{2}}+\frac{E_{\mathrm{NV}}\left(S_{+}^{2}+S_{-}^{2}\right)}{2 \hbar^{2}}-\gamma_{\mathrm{e}} \frac{\mathbf{B} \cdot \mathbf{S}}{\hbar},
$$

where $D_{\mathrm{NV}}=2 \pi \times 2.87 \mathrm{GHz}$ is the zero field splitting, $E_{\mathrm{NV}} \ll D_{\mathrm{NV}}$ is a strain-induced splitting, $\gamma_{\mathrm{e}}=$ 


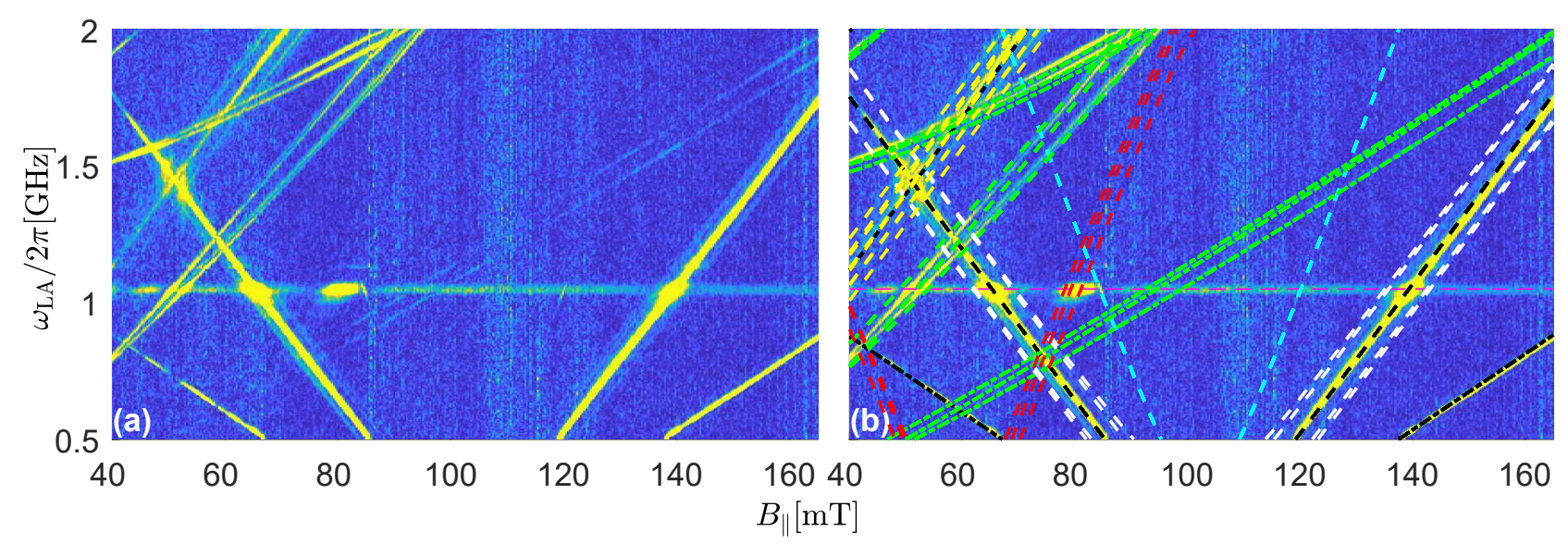

FIG. 2: Big picture ODMR plot. Side coils were tuned to compensate the misalignment at $B_{\|}=38 \mathrm{mT}$. Dashed black lines (color online) correspond to $T_{\alpha}^{-1,0}$ transition, symmetrical about the $m_{\mathrm{S}}=-1$ and $m_{\mathrm{S}}=0$ levels anticrossing at $102 \mathrm{mT}$. White lines are due to the hyperfine interaction of $\mathrm{NV}^{-}$with first shell $13 \mathrm{C}$ isotope [12, 20 24]. Black dash-dotted lines represent multiphoton processes at half the frequency of $T_{\alpha}^{-1,0}$. Cyan line, only visible in the data at the vicinity of the MW resonator frequency, represents the double Larmor line of $T_{\alpha}^{-1,0}$, interpreted as flip-flip (see Fig. 4). Green dashed lines represent the $T_{\beta, \gamma, \delta}^{-1,+1}$ transitions of the three $\mathrm{NV}^{-}$vectors not parallel to the bias magnetic field. The top (bottom) dash-dotted green lines represent the double-photon transitions $T_{\beta, \gamma, \delta}^{-1,0}\left(T_{\beta, \gamma, \delta}^{-1,+1}\right)$. The red lines (visible only near the resonator resonance) correspond to the difference between the transitions $T_{\beta, \gamma, \delta}^{-1,+1}$ and $T_{\alpha}^{-1,0}$, interpreted as spin flip-flop (see Fig. 5 ). The yellow lines correspond to the P1 hyperfine-split $m_{\mathrm{S}}=-1 / 2$ to $m_{\mathrm{S}}=+1 / 2$ transition (see Fig. 6), while the parallel black dotted line in their center represents the double-photon $T_{\alpha}^{-1,+1}$ transition. The magenta line of the MW resonator is of unclear origin [25], however the signal enhancement of other resonances in the vicinity of the resonator frequency might be attributed to a higher effective MW power.

$2 \pi \times 28.03 \mathrm{GHzT}^{-1}$ is the electron spin gyromagnetic ratio, B is the applied magnetic field, $\mathbf{S}=S_{x} \hat{\mathbf{x}}+S_{y} \hat{\mathbf{y}}+S_{z} \hat{\mathbf{z}}$ is the spin $S=1$ angular momentum vector operator and $S_{ \pm}=S_{x} \pm i S_{y}$. Under continuous laser excitation, $\mathrm{NV}^{-}$is polarized to the spin state having magnetic quantum number $m_{\mathrm{S}}=0$, which has a slightly brighter photoluminescence (PL) 1]. Introducing MW irradiation at frequency $\omega_{\mathrm{LA}} / 2 \pi$ resonant with $m_{\mathrm{S}}=0$ to $m_{\mathrm{S}}= \pm 1$ transition reduces the spin polarization and $\mathrm{PL}$, allowing optical detection of the magnetic resonance (ODMR). We will use a nomenclature of $T_{v}^{i, j}$ to indicate a resonant transition in $\mathrm{NV}^{-}$center parallel to vector $v \in\{\alpha, \beta, \gamma, \delta\}$ (see inset in Fig. 1) from state $i$ to state $j$, where $i, j \in\{-1,0,+1\}$. Both $i$ and $j$ states are eigenstates of the Hamiltonian $\mathcal{H}_{\mathrm{NV}}$ (11), where $i$ and $j$ indicate the magnetic quantum number $m_{\mathrm{S}}$ when $\left[\mathcal{H}_{\mathrm{NV}}, S_{z}\right]=0$ (i.e. when the externally applied magnetic field $\mathbf{B}$ is parallel to the symmetry axis of the $\mathrm{NV}^{-}$center, and straininduced splitting is disregarded). A typical ODMR plot is shown in Fig. 2

Our ODMR plots show not only the $\mathrm{NV}^{-}$resonances, but also other defects. Among them - nitrogen 14 (nuclear spin 1) substitution defect (P1) in diamond [24, 28], which has four locally stable configurations. In each configuration a static Jahn-Teller distortion [29] occurs, and an unpaired electron is shared by the nitrogen atom and by one of the four neighboring carbon atoms, which are positioned along one of the lattice directions $\langle 111\rangle[14,3038]$. The transition frequencies are calcu- lated (see Figs. 2, 5] and 6) by numerically diagonalizing the $\mathrm{P} 1$ spin Hamiltonian $\mathcal{H}_{\mathrm{P} 1}$ [12, 39] using the following parameters: nitrogen 14 nuclear gyromagnetic ratio $\gamma_{\mathrm{n}}=2 \pi \times 3.0766 \mathrm{MHz}$, nitrogen 14 quadrupole coupling $Q_{\mathrm{P} 1}=-2 \pi \times 3.97 \mathrm{MHz}$, longitudinal hyperfine coupling $A_{\mathrm{P} 1, \|}=2 \pi \times 114 \mathrm{MHz}$ and transverse hyperfine coupling $A_{\mathrm{P} 1, \|}=2 \pi \times 81.3 \mathrm{MHz}$.

Type Ib HPHT single crystal [110] grown diamond with $<200$ ppm nitrogen concentration (same as in [12]) was laser-cut, polished, irradiated with $2.8 \mathrm{MeV}$ electrons at a doze of $8 \times 10^{18} \mathrm{e} / \mathrm{cm}^{2}$, annealed for $2 \mathrm{~h}$ at $900 \mathrm{C}$ and boiled for $1 \mathrm{~h}$ in equal mixture of Perchloric, Sulfuric and Fuming Nitric acids, resulting with a fluorescent-count measured $\mathrm{NV}^{-}$concentration of $n_{\mathrm{S}}=3.25 \times 10^{17} \mathrm{~cm}^{-3}$. The $\mathrm{NV}^{-}: \mathrm{P} 1$ ratio is estimated from electron spin resonance (ESR) data to be $1: 3$ (see Fig. 31 [12]. The diamond is oriented at room temperature to have the $\alpha([1 \overline{1} \overline{1}])$ axis coinciding with the main coil axis and the (110) surface orthogonal to the 2nd coil. The diamond wafer is glued to a sapphire substrate carrying a $1.05 \mathrm{GHz}$ superconducting spiral resonator (see Fig. 11). The resonator is not used in this experiment. All measurements are performed at $3.5 \mathrm{~K}$. Throughout the paper, the diamond misalignment is fit by azimuthal $\theta=2.86^{\circ}$ and polar $\phi=1.71^{\circ}$ angles with respect to the coordinate system defined by the axial directions of the 1st, 2 nd and the main superconducting coils (see Fig. 1). A $637 \mathrm{~nm}$ red laser is used to excite the $\mathrm{NV}$ centers at the $\mathrm{NV}^{-}$zerophonon line (ZPL), rather than the more commonly used 


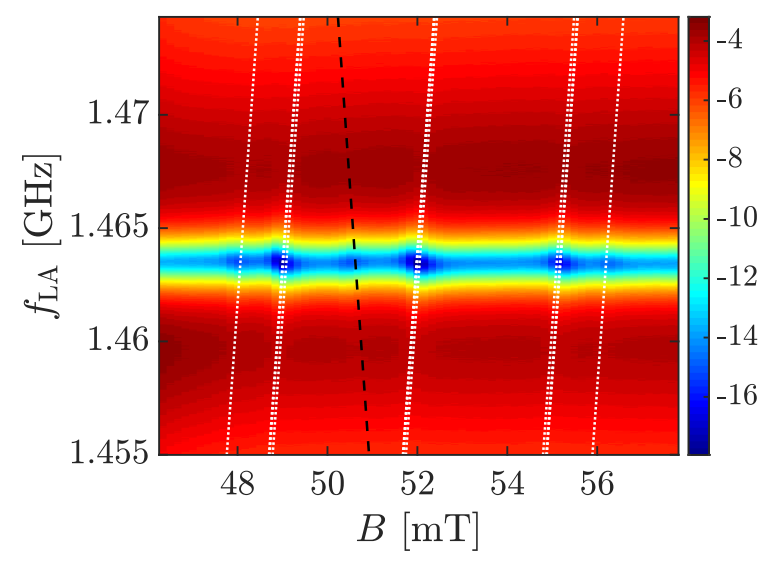

FIG. 3: MW cavity ESR measurement without laser polarisation, as reported in reference [12], with 5 resolved P1 resonances fit by white dotted lines [see Eq. (3) in [12]] and the $\mathrm{NV}^{-}$parallel to magnetic field $m_{\mathrm{S}}=0$ to $m_{\mathrm{S}}=-1$ resonance fit by a black dashed line [see Eq. (1) in [12]]. The color represents reflection coefficient $S_{11}$ in dB units, and the superconducting resonator is different, having a frequency of $1.463 \mathrm{GHz}$. The diamond orientation is similar to the one in the current work, and the fit parameters are as in caption of Fig.2. of [12]. The comparable cavity frequency shift as result of $\mathrm{NV}^{-}$resonance $\left(1 / 4\right.$ of total $\mathrm{NV}^{-}$ensemble) and the weakest of $\mathrm{P} 1$ resonances (1/12 of the $\mathrm{P} 1$ ensemble) results with an estimate of $\mathrm{NV}^{-}$to $\mathrm{P} 1$ concentration ratio of $1: 3$.

$532 \mathrm{~nm}$, in order to reduce sample heating. Despite the expected photoionization to the neutrally charged NV state, no trace of these defects was recorded in the ODMR scans. The returning PL light passes through a beam splitter and a $700 \mathrm{~nm}$ long pass filter before measurement with a reverse-biased photo-diode (PD). MW loop antenna is connected directly to a synthesizer, $100 \%$ amplitude modulated by a $151 \mathrm{~Hz}$ sine wave. The PD signal is demodulated by a lock-in amplifier [40], and the resulting amplitude is recorded. In all the two dimensional scans the magnetic field is scanned first, slowly enough to avoid hysteresis.

\section{SAME SPIN-SPIN INTERACTION}

For $\mathrm{NV}^{-}$aligned closely along the magnetic field direction (having a misalignment angle $|\psi| \ll 1 \mathrm{rad}$ ), a ground state levels anticrossing (GSLAC) between the states $m_{\mathrm{S}}=0$ and $m_{\mathrm{S}}=-1$ occurs at $D_{\mathrm{NV}} / \gamma_{\mathrm{e}}=102.4 \mathrm{mT}$. The resonance angular frequency $\omega_{\mathrm{a}}$ and the effective transverse drive amplitude $\omega_{\mathrm{t}}$ are given [15, 41] by

$$
\begin{gathered}
\omega_{\mathrm{a}}=\omega_{\mathrm{a} 0} \sqrt{1+\eta^{2}}, \\
\omega_{\mathrm{t}}=\omega_{\mathrm{LA} 1} \eta / \sqrt{1+\eta^{2}},
\end{gathered}
$$

where $\omega_{\mathrm{a} 0}=\sqrt{2} D_{\mathrm{NV}}|\psi|, \omega_{\mathrm{LA} 1}$ is the MW loop antenna drive amplitude, and the dimensionless detuning parameter $\eta$ is given by $\eta=\left(D_{\mathrm{NV}}-\gamma_{\mathrm{e}} B\right) / \omega_{\mathrm{a} 0}$. The signal
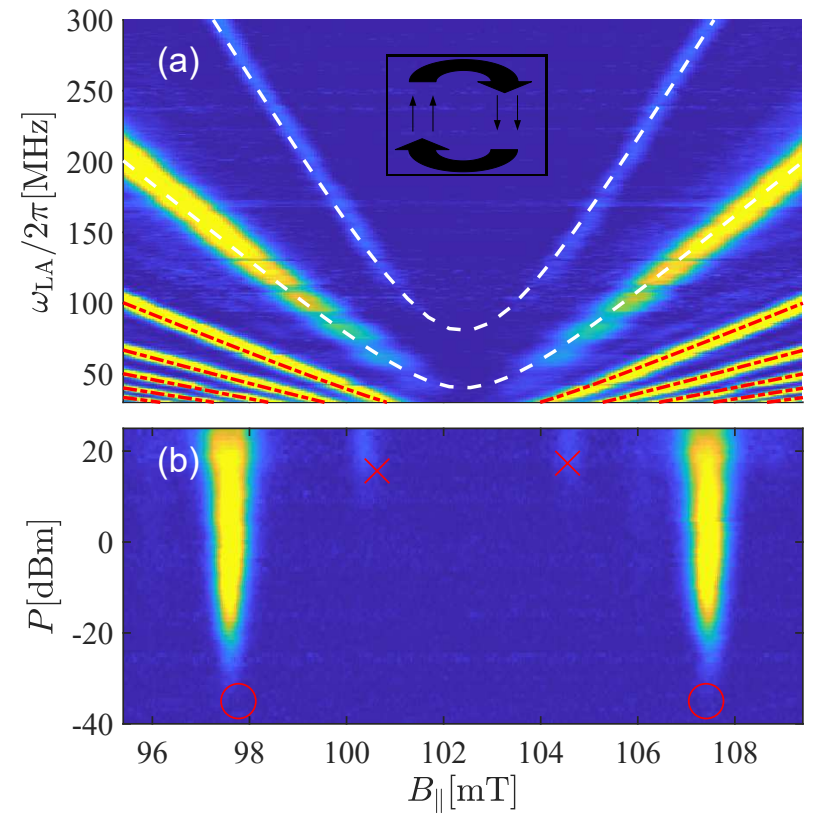

FIG. 4: Double Larmor frequency ODMR. (a) White dashed lines represent fit of first and second Larmor frequencies calculated according to Eq. (2) with $\psi=0.8^{\circ}$, incorporating both the diamond misalignment and side coils correction. The red dash-dotted lines are calculated as multi-photon resonances by Eq. (4). Alternatively, the $1 / m$ hyperbolas might be attributed to higher harmonics of the synthesizer, enhanced by amplitude modulation. Inset: schematic representation of spin flip-flip process. (b) MW power dependency of the ODMR signal at $\omega_{\mathrm{LA}}=2 \pi \times 145 \mathrm{MHz}$. Circles mark the beginning of first hyperbola visibility $\left(P_{1}\right)$, indicating $2 \%$ of the maximal recorded signal. Crosses mark the expected beginning of the second hyperbola visibility, given by $R_{2} P_{1}$ [see Eq. (5)]. The signal reduction at high MW power is due to lock-in saturation artifact; throughout our measurements higher laser and MW powers lead to higher ODMR contrast.

is recognizable in the ODMR scan as a hyperbola disappearing near the GSLAC, due to spin states mixing and subsequent inefficient MW depolarization required for the ODMR contrast.

Dipolar coupling gives rise to an absorption resonance at an angular frequency $2 \omega_{\mathrm{a}}$ [second hyperbola, see Fig. 4(a)], due to spins flip-flip. Fractional hyperbolas at angular frequencies $\omega_{\mathrm{a}} / l$ for integer $l$ are attributed to multi-photon processes or higher harmonics in the excitation signal. The Bloch-Siegert shift corresponding to the l'th multi-photon processes is given by [see Eq.(6.378) of [42]]

$$
\omega_{\mathrm{BS}, l}=\sum_{l^{\prime} \neq l} \frac{\omega_{\mathrm{t}}^{2}\left(J_{l^{\prime}}\left(\frac{\omega_{\mathrm{S} 1}}{\omega_{\mathrm{LA}}}\right)+J_{l^{\prime}+2}\left(\frac{\omega_{\mathrm{S} 1}}{\omega_{\mathrm{LA}}}\right)\right)^{2}}{2\left(l-l^{\prime}\right) \omega_{\mathrm{LA}}}
$$

where $\omega_{\mathrm{S} 1}$ is longitudinal excitation amplitudes. The shift is too small to be resolved in our measurements, 
hence we conclude that $\omega_{\mathrm{LA} 1}<1 \mathrm{MHz}$, and the ODMR linewidth is caused not by power broadening, but by increased $\mathrm{NV}^{-}$transverse relaxation or by inhomogeneous broadening.

The ratio between the second and first hyperbolas strength at the effective magnetic field $B_{\mathrm{a}}=\omega_{\mathrm{a}} / \gamma_{\mathrm{e}}$ is given by 43 46]

$$
R_{2}=\frac{2}{3}\left\langle(\Delta B)^{2}\right\rangle_{a v} / B_{\mathrm{a}}^{2},
$$

where, for disordered defects location, the azimuthally averaged local field is $\left\langle(\Delta B)^{2}\right\rangle_{a v}=\pi \mu_{0}^{2} \hbar^{2} \gamma_{\mathrm{e}}^{2} S(S+$ 1) $\Sigma_{j} r_{i j}^{-6}$ and $r_{i j}$ is the distance between defects $i$ and $j$. For diluted diamond lattice $\Sigma_{j} r_{i j}^{-6}=r_{\text {eff }}^{-6}$, where we calculate $r_{\text {eff }}=0.907 / \sqrt[3]{\rho_{\mathrm{NV}}}$ and $\rho_{\mathrm{NV}}=3.25 / 4 \times 10^{17} \mathrm{~cm}^{-3}$ is the density of $\mathrm{NV}^{-}$parallel to one of the crystallographic vectors. A quantitative comparison of first and second hyperbolas visibility is given in Fig. 4(b), and a good agreement to optically determined NV concentration is found.

\section{DIFFERENT SPIN-SPIN INTERACTION}

We observe dipolar spin flip-flop lines between ensembles of different $\mathrm{NV}^{-}$orientations, specifically between the $T_{\alpha}^{-1,0}$ transition of the $\mathrm{NV}^{-}$parallel to the main coil magnetic field and $T_{\beta, \gamma, \delta}^{-1,+1}$ transitions of the remaining three $\mathrm{NV}^{-}$orientations [see Fig. [5(d)]. The signal visibility of $T_{\beta, \gamma, \delta}^{-1,+1}$ is low as compared to $T_{\alpha}^{-1,0}$ [see Fig. 55(c)], and therefore the flip-flop ODMR signal is attributed mostly to the latter. Applying magnetic field $B_{\perp}$ in the [110] direction by increasing the current in the 2nd coil lifts the geometric degeneracy of the three non-parallel $\mathrm{NV}^{-}$vectors [see Fig. [5(b)]. The visibility of $T_{\beta}^{-1,+1}$ is much smaller than the visibility of $T_{\gamma, \delta}^{-1,+1}$, probably due to the orientation with respect to the MW antenna, and the corresponding flip-flop signal was not detected. The relative strength of the flip-flop signal [Fig. [5(f)] is comparable [47, p. 357] to the same spin flip-flip case [Fig. 4 (b)] (i.e. both signals become detectable at an input power of $10 \mathrm{dBm})$.

In addition, faint diagonal lines circa $53 \mathrm{mT}$ are attributed to a spin-flip process between the parallel $\mathrm{NV}^{-}$and an additional electronic spin- $1 / 2$ ensemble [Fig. [5(g)]. A suitable candidate is the P1 ensemble, though the signal strength does not allow reliable fit of the hyperfine lines. The expected P1 concentration is comparable to $\mathrm{NV}^{-}$, yet the $\mathrm{NV}^{-}-\mathrm{P} 1$ flip-flop is hardly registered.

A possible explanation is an efficient hyperpolarization of the $\mathrm{P} 1$ centers as compared to the nonparallel $\mathrm{NV}^{-}$centers, leading to a lower flip-flop probability. The reduced non-parallel $\mathrm{NV}^{-}$hyperpolarization may in turn be explained by spin flips during optical excitation-relaxation cycles or during photoionization and electron re-trapping. Yet a different explanation could be a photo-ionisation of the substitutional nitrogen, effectively reducing the concentration of the paramagnetic P1 centers [48, 49]. However, the data of Fig. 6] suggests that the P1 concentration is significant enough to alter the $\mathrm{NV}^{-} \mathrm{ODMR}$ even when the P1 frequency is detuned far from the $\mathrm{NV}^{-}$resonance.

\section{ADDITIONAL RESULTS}

In low frequency region all observed ODMR lines are modulated with a pattern having a characteristic beating frequency given by $f_{\mathrm{a}}=20.4 \mathrm{MHz}$ (Fig. 7). The beating can be attributed to strain coupling to bulk acoustic standing waves in the diamond wafer $[50-52]$. However, the speed of sound of $2 f_{\mathrm{a}} t=20.4 \mathrm{Km} / \mathrm{s}$, which is derived from the measured beating frequency $f_{\mathrm{a}}$ and the thickness of the diamond wafer $t=0.5 \mathrm{~mm}$, is about $13 \%$ higher than the values reported in [51, 53, 54].

The ODMR data shown in Fig. 8 for the frequency range of $10-13 \mathrm{MHz}$ exhibits a complex modulation pattern. Three nuclear spins have been considered to explain the data. The nuclear spin 1 of nitrogen 14 of P1 defects is ruled out since its transition frequency is not expected to significantly change near $\mathrm{NV}^{-}$GSLAC, whereas the data exhibits modulation pattern of arcs symmetric around $102 \mathrm{mT}$. The spin 1 of nitrogen 14 of the NVdefects is ruled out since its hyperfine coupling is too weak. First shell $13 \mathrm{C}$ has both sufficiently strong hyperfine coupling and its transition frequency strongly varies near $102 \mathrm{mT}$, however the high symmetry of the data is not compatible with $13 \mathrm{C}$ energy levels. Perplexedly, the arcs in the figure can be fitted by a single curve, stretched along the magnetic field axis by an integer factor (see caption of Fig. 8).

\section{DISCUSSION}

We report of optical detection of driven spin flip-flop, yet many questions remain unanswered with regard to the strength of perceived signal. For bath-control applications, of utter importance is the poor visibility of $\mathrm{NV}^{-}$P1 flip-flop line, which suggests high efficiency of P1 optical DNP process. With regards to $\mathrm{NV}^{-}-\mathrm{NV}^{-}$flip flops, the $\beta$ direction stands out, with both poor visibility of $T_{\beta}^{-1,+1}$ transition and the lack of detectable flip-flop with $T_{\alpha}^{-1,0}$. In addition, no flip-flip lines at $2 T_{\beta, \gamma, \delta}^{-1,+1}$ were detected. Further studies altering the relative orientations of the diamond, bias magnetic field and MW excitation may provide further insight regarding the strength of the various flip-flop lines, particularly in relation to the well understood flip-flip line strength. The strength of the flip-flop lines may be measured to optimize the efficiency of applied hyperpolarization protocols - i.e., after efficient hyperpolarization there are more aligned spins and less misaligned, hence flip-flop line is suppressed and the flip-flip line is emphasized. 


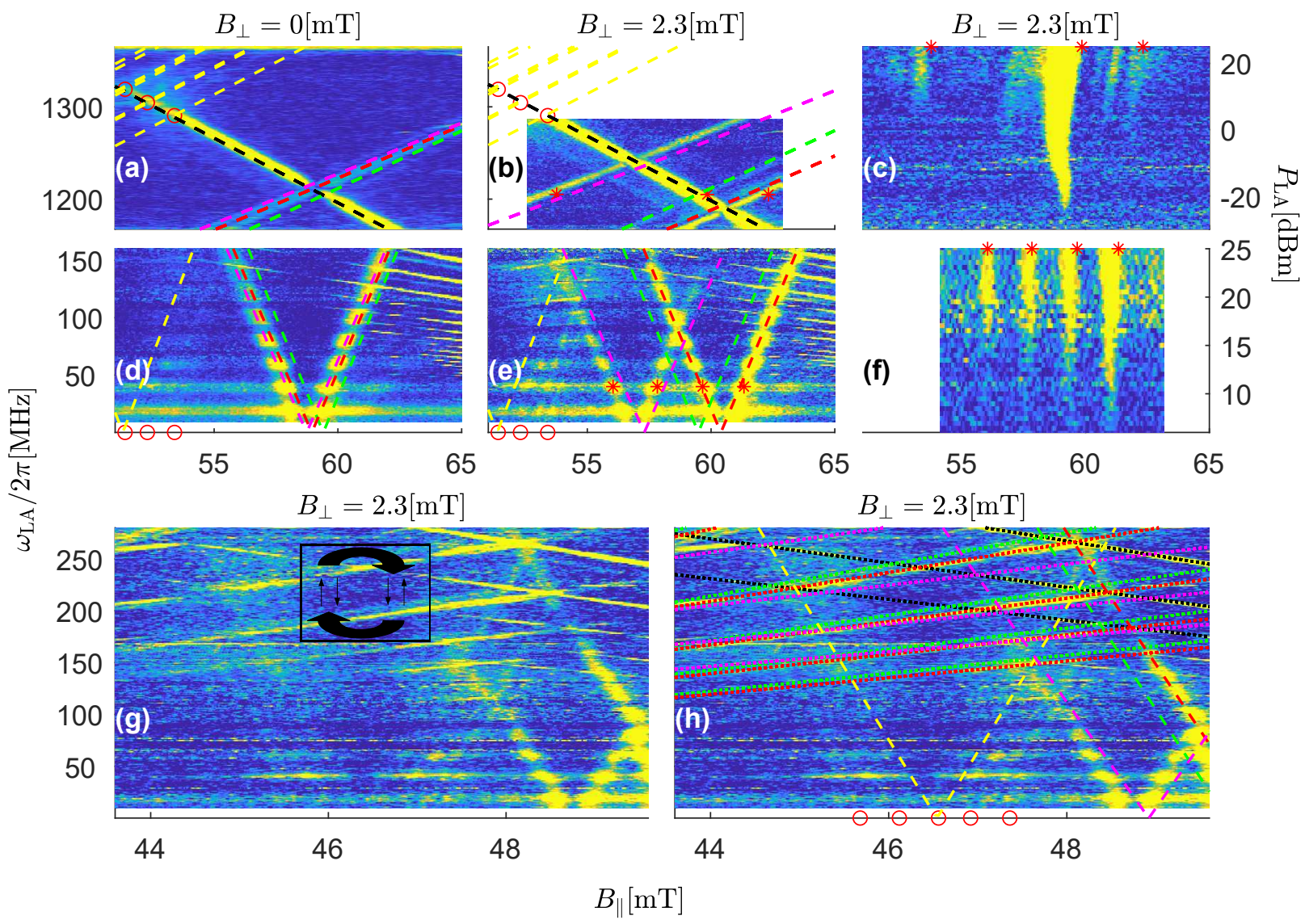

FIG. 5: Flip-flop ODMR. (a) Black/green/red/magenta (color online) are the $T_{\alpha}^{-1,0} / T_{\beta}^{-1,+1} / T_{\gamma}^{-1,+1} / T_{\delta}^{-1,+1}$ transitions calculated according to Eq. (1). Yellow lines are P1 hyperfine transitions calculated as in Fig. 6. The side coils magnetic field is set to compensate the sample misalignment at the main coil field of $B_{\|}=59 \mathrm{mT}$, as validated by measuring the degeneracy of $T_{\beta, \gamma, \delta}^{-1,0}$ transitions (not shown). (b) Same as (a), but the 2nd coil magnetic field is increased by $B_{\perp}=2.3 \mathrm{mT}$, lifting the degeneracy of the $\mathrm{NV}^{-}$parallel to $\beta, \gamma, \delta$ vectors. (c) MW power scan along the $1210 \mathrm{MHz}$ line in the otherwise similar conditions to (b). Red asterisks in (b) and (c) mark similar points in the parameter space. (d) Taken in the same magnetic conditions as (a). Green/red/magenta lines are the difference between the corresponding color lines and the black line in (a). Yellow line is the difference between the P1 $m_{\mathrm{S}}=+1 / 2$ to $m_{\mathrm{S}}=-1 / 2$ transition with nuclear spin number $m_{\mathrm{I}}=0$ and the black line in (a), while the red circles mark the intersections between the yellow lines and the black line in (a). (e) is to (b) as (d) is to (a), and (f) is to (e) as (c) is to (b) but along the $40 \mathrm{MHz}$ line. (g) and (h) show the same data taken in the magnetic conditions of (e). The dashed lines in (h) correspond to the dashed lines in (e) of the same color. Dotted lines in (h) correspond to the dashed lines of the same color in (b) divided by an integer, representing multiphoton or higher harmonics signal. The red circles are calculated as in (e). Inset in $(\mathrm{g})$ is a schematic representation of the spin flip-flop.

\section{ACKNOWLEDGMENTS}

We thank Aharon Blank, Amit Finkler and Nir Bar Gill for fruitful discussions. This work was supported by the Israel science foundation, any by the Israel ministry of science.
[1] M. W. Doherty, N. B. Manson, P. Delaney, F. Jelezko, J. Wrachtrup, and L. C. Hollenberg, Physics Reports 528, 1 (2013).

[2] L. Rondin, J. Tetienne, T. Hingant, J. Roch, P. Maletinsky, and V. Jacques, Reports on Progress in Physics 77, 056503 (2014).
[3] S. Dhomkar, J. Henshaw, H. Jayakumar, and C. A. Meriles, Science advances 2, e1600911 (2016).

[4] X. Zhu, S. Saito, A. Kemp, K. Kakuyanagi, S.-i. Karimoto, H. Nakano, W. J. Munro, Y. Tokura, M. S. Everitt, K. Nemoto, et al., Nature 478, 221 (2011).

[5] Y. Kubo, C. Grezes, A. Dewes, T. Umeda, J. Isoya, 

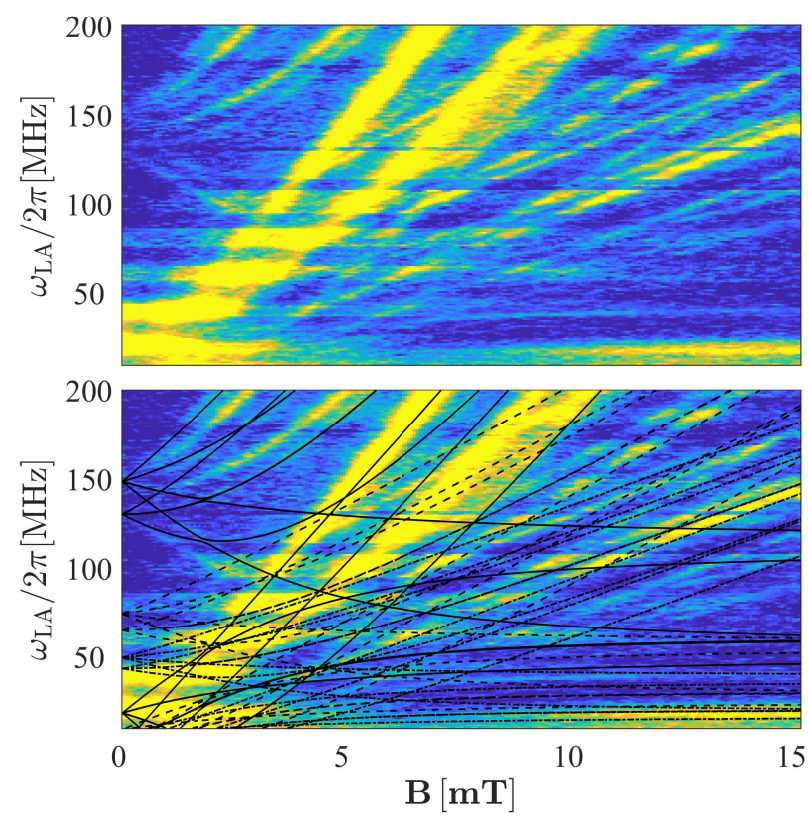

FIG. 6: P1 ODMR. Black solid/dashed/dot-dashed lines are some of the first/second/third order P1 transitions. The magnetic field orientation in the fit is the same as in Fig. 5] without current in the side coils.

H. Sumiya, N. Morishita, H. Abe, S. Onoda, T. Ohshima, et al., Physical review letters 107, 220501 (2011).

[6] R. Amsüss, C. Koller, T. Nöbauer, S. Putz, S. Rotter, K. Sandner, S. Schneider, M. Schramböck, G. Steinhauser, H. Ritsch, et al., Phys. Rev. Lett. 107, 060502 (2011), URL http://link.aps.org/doi/10.1103/PhysRevLett.107.060502

[7] C. Grezes, B. Julsgaard, Y. Kubo, M. Stern, T. Umeda, J. Isoya, H. Sumiya, H. Abe, S. Onoda, T. Ohshima, et al., Physical Review X 4, 021049 (2014).

[8] J. D. Breeze, E. Salvadori, J. Sathian, N. M. Alford, and C. W. Kay, Nature 555, 493 (2018).

[9] A. Bermudez, F. Jelezko, M. Plenio, and A. Retzker, Physical review letters 107, 150503 (2011).

[10] N. Bar-Gill, L. M. Pham, C. Belthangady, D. Le Sage, P. Cappellaro, J. Maze, M. D. Lukin, A. Yacoby, and R. Walsworth, Nature communications 3, 1 (2012).

[11] N. Bloembergen, S. Shapiro, P. Pershan, and J. Artman, Physical Review 114, 445 (1959).

[12] N. Alfasi, S. Masis, O. Shtempluck, and E. Buks, Phys. Rev. B 99, 214111 (2019).

[13] S. Armstrong, L. J. Rogers, R. L. McMurtrie, and N. B. Manson, Physics Procedia 3, 1569 (2010).

[14] H.-J. Wang, C. S. Shin, S. J. Seltzer, C. E. Avalos, A. Pines, and V. S. Bajaj, Nature communications 5, 4135 (2014).

[15] S. Masis, N. Alfasi, R. Levi, O. Shtempluck, and E. Buks, Phys. Rev. A 100, 013852 (2019).

[16] I. Waller, Zeitschrift für Physik 79, 370 (1932).

[17] M. M. Dorio, Multiple electron resonance spectroscopy (Springer Science \& Business Media, 2012).

[18] C. Belthangady, N. Bar-Gill, L. M. Pham, K. Arai,
D. Le Sage, P. Cappellaro, and R. L. Walsworth, Physical review letters 110, 157601 (2013).

[19] E. Kamp, B. Carvajal, and N. Samarth, Physical Review B 97, 045204 (2018).

[20] S. Felton, A. Edmonds, M. Newton, P. Martineau, D. Fisher, D. Twitchen, and J. Baker, Physical Review B 79, 075203 (2009).

[21] N. Mizuochi, P. Neumann, F. Rempp, J. Beck, V. Jacques, P. Siyushev, K. Nakamura, D. Twitchen, H. Watanabe, S. Yamasaki, et al., Physical review B 80, 041201 (2009).

[22] M. Simanovskaia, K. Jensen, A. Jarmola, K. Aulenbacher, N. Manson, and D. Budker, Physical Review B 87, 224106 (2013).

[23] E. Kamp, B. Carvajal, and N. Samarth, Physical Review B 97, 045204 (2018).

[24] J. Loubser and J. van Wyk, Reports on Progress in Physics 41, 1201 (1978).

[25] N. Alfasi, S. Masis, R. Winik, D. Farfurnik, O. Shtempluck, N. Bar-Gill, and E. Buks, Physical Review A 97, 063808 (2018).

[26] P. Ovartchaiyapong, K. W. Lee, B. A. Myers, and A. C. B. Jayich, arXiv:1403.4173 (2014).

[27] E. MacQuarrie, T. Gosavi, N. Jungwirth, S. Bhave, and G. Fuchs, Physical review letters 111, 227602 (2013).

[28] R. Cook and D. Whiffen, Proceedings of the Royal Society of London A: Mathematical, Physical and Engineering Sciences 295, 99 (1966).

[29] W. Smith, P. Sorokin, I. Gelles, and G. Lasher, Physical Review 115, 1546 (1959).

[30] S. Takahashi, R. Hanson, J. van Tol, M. S. Sherwin, and D. D. Awschalom, Physical review letters 101, 047601 (2008).

[31] R. Hanson, F. Mendoza, R. Epstein, and D. Awschalom, Physical review letters 97, 087601 (2006).

[32] R. Hanson, V. Dobrovitski, A. Feiguin, O. Gywat, and D. Awschalom, Science 320, 352 (2008).

[33] D. A. Broadway, J. D. Wood, L. T. Hall, A. Stacey, M. Markham, D. A. Simpson, J.-P. Tetienne, and L. C. Hollenberg, arXiv:1607.04006 (2016).

[34] J. Shim, B. Nowak, I. Niemeyer, J. Zhang, F. Brandao, and D. Suter, arXiv:1307.0257 (2013).

[35] B. Smeltzer, L. Childress, and A. Gali, New Journal of Physics 13, 025021 (2011).

[36] C. S. Shin, M. C. Butler, H.-J. Wang, C. E. Avalos, S. J. Seltzer, R.-B. Liu, A. Pines, and V. S. Bajaj, Physical Review B 89, 205202 (2014).

[37] H. Clevenson, E. H. Chen, F. Dolde, C. Teale, D. Englund, and D. Braje, Phys. Rev. A 94, 021401 (2016).

[38] D. Schuster, A. Sears, E. Ginossar, L. DiCarlo, L. Frunzio, J. Morton, H. Wu, G. Briggs, B. Buckley, D. Awschalom, et al., Physical review letters 105, 140501 (2010).

[39] A. Cox, M. Newton, and J. Baker, Journal of Physics: Condensed Matter 6, 551 (1994).

[40] S. V. Anishchik and K. L. Ivanov, Physical Review B 96, 115142 (2017).

[41] S. Masis, Dipolar interactions between dense spinensembles in diamo

[42] E. Buks, Quantum mechanics - Lecture Notes (http://buks.net.technion.ac.il/teaching/, 2020), URL http://buks.net.technion.ac.il/teaching/

[43] A. Anderson, Physical Review 125, 1517 (1962).

[44] H. Cheng, Physical Review 124, 1359 (1961).

[45] L. Broer, Physica 10, 801 (1943). 


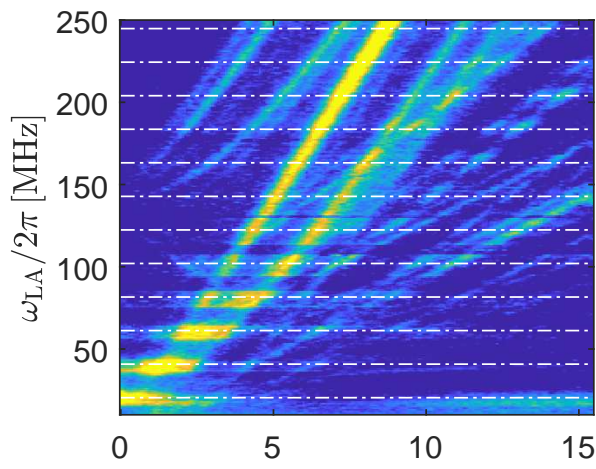

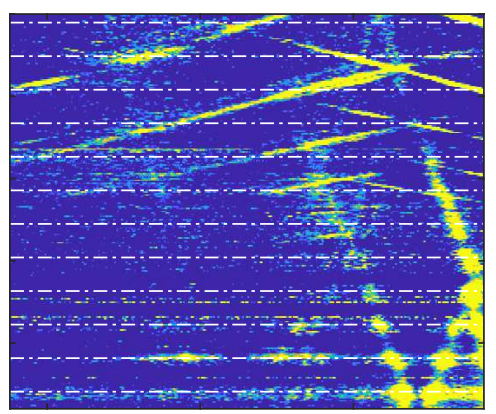

45
50 $B[\mathrm{mT}]$

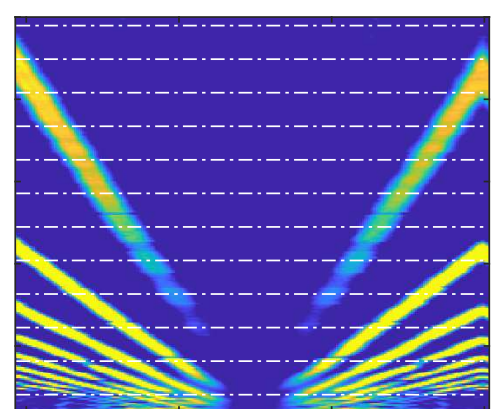

95

100

105

110

FIG. 7: Acoustic resonator. ODMR from Figs. 6. 4 and 5. White dashed lines plotted at $n \times f_{\mathrm{a}}=20.4 \mathrm{MHz}$ for integer $n$.

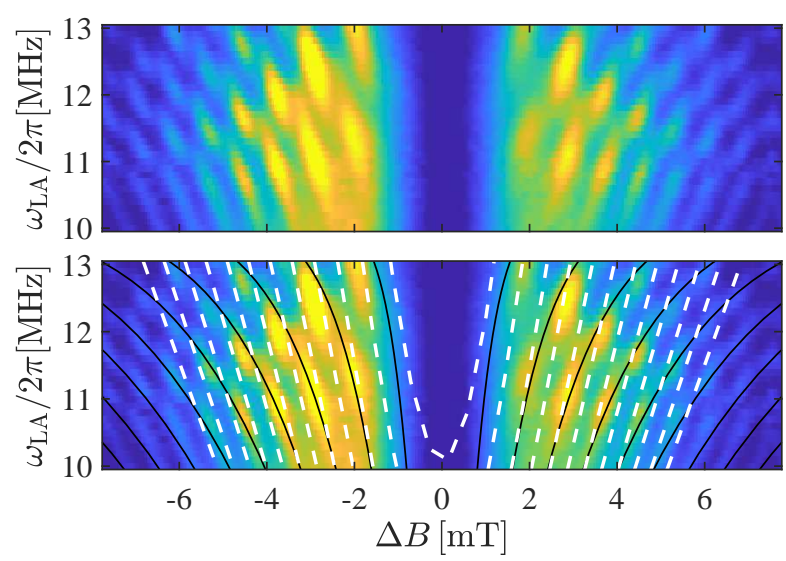

FIG. 8: Low frequency ODMR near GSLAC. The black lines represent empirical relation between frequency $f$ and magnetic field detuning from the GSLAC $\Delta B$ given by $f=f_{\operatorname{arc}}\left|\tanh \left(\Delta B / n B_{\text {arc }}\right)\right|$, with $f_{\text {arc }}=13.9 \mathrm{MHz}, B_{\text {arc }}=$ $0.89 \mathrm{mT}$ and an integer $n$. White dashed lines are integer fractions of $\omega_{\mathrm{a}}$, as calculated by Eq. (2) for the same $\theta$ as in Fig. 4

[46] J. Daycock and G. P. Jones, Journal of Physics C: Solid State Physics 2, 998 (1969).

[47] A. Abragam and M. Goldman (1982).

[48] M. Doherty, C. A. Meriles, A. Alkauskas, H. Fedder, M. J. Sellars, and N. B. Manson, Physical Review X 6, 041035 (2016).

[49] S. C. Lawson, D. Fisher, D. C. Hunt, and M. E. Newton, Journal of Physics: Condensed Matter 10, 6171 (1998).

[50] D. A. Golter, T. Oo, M. Amezcua, K. A. Stewart, and H. Wang, Physical review letters 116, 143602 (2016).

[51] N. Nakamura, H. Ogi, T. Ichitsubo, M. Hirao, N. Tatsumi, T. Imai, and H. Nakahata, Journal of applied physics 94, 6405 (2003).

[52] P. Ovartchaiyapong, L. Pascal, B. Myers, P. Lauria, and A. B. Jayich, Applied Physics Letters 101, 163505 (2012).

[53] H. McSkimin and W. Bond, Physical Review 105, 116 (1957).

[54] H. McSkimin and P. Andreatch Jr, Journal of Applied
Physics 43, 2944 (1972). 